\title{
The asymmetrical visual evoked potential to pattern reversal in one half field and its significance for the analysis of visual field defects
}

\author{
L. D. BLUMHARDT, G. BARRETT, AND A. M. HALlidAY \\ From the Medical Research Council, Institute of Neurology, National Hospital, Queen Square, London, WCI
}

SUMMARY The consistency of the major positive component $(\overline{\mathrm{P} 100})$ of the full-field pattern-reversal response provides a clinically valuable and objective means of detecting visual field defects. Its normally symmetrical distribution about the midline of the occipital scalp results from the summation of two highly asymmetric half-field responses, each of which shows the positive component well lateralised with a widespread distribution on the ipsilateral side. Stimulation of each eye in patients with bitemporal and homonymous hemianopias results in two characteristic patterns of asymmetry, named 'crossed' and 'uncrossed' respectively, in which the major positivity is consistently recorded on the side ipsilateral to the preserved half field. Recordings from a patient after occipital lobectomy confirm the authors' previous suggestion that although the major positive component is recorded from the ipsilateral scalp the typical asymmetric half-field response is generated in the contralateral hemisphere.

The wide variability of the cortical evoked potential to flash stimulation in healthy subjects has led some authors to conclude that recording the visual evoked response has little value in clinical diagnosis (Oosterhuis et al., 1969). The use of unstructured flash stimuli in these early studies made it difficult to define the limits of the normal response. In these circumstances objective evidence of even dense hemianopic field defects was relatively hard to obtain (Vaughan et al., 1963; Kooi et al., 1965; Oosterhuis et al., 1969).

In contrast, the consistency of the early components of the transient cortical response to patternreversal stimulation has allowed reliable intersubject comparisons to be made. The most prominent and constant component is the major positive wave $(\overline{P 100})$, which has a narrow latency range in healthy subjects and has thus proved of considerable clinical value in detecting and confirming delayed responses in demyelinating optic neuropathy (Halliday et al., 1972, 1973). A further advantage of a pattern stimulus is that it allows the apparent separation of the hemisphere responses, as demonstrated by Cobb and Morton (1970), but the choice of montage and reference electrode is critical in

Address for reprints: Dr A. M. Halliday, Medical Research Council, Institute of Neurology, National Hospital, Queen Square, London, WC1 the interpretation of the topography of the response (Michael and Halliday, 1971; Barrett et al., 1976b).

We have recently described the paradoxical lateralisation of the half-field response to patternreversal stimulation (Barrett et al., 1976a). The major positive component ( $\overline{\mathrm{P} 100})$, which has a latency of about $105 \mathrm{~ms}$, was consistently recorded from the electrodes ipsilateral to the half field stimulated, while the activity recorded contralaterally was usually much smaller and distinctly different in character (Blumhardt et al., 1977a). Recordings from patients with hemianopic field defects confirm the findings in healthy subjects. The understanding of this consistently asymmetrical half-field response is essential for the interpretation of pattern-evoked potentials recorded from patients with visual pathway pathology.

\section{Methods}

Nine healthy persons were used for the study of the normal half-field response. Chlorided silver cup electrodes $9 \mathrm{~mm}$ in diameter were fixed to the scalp with collodion. The montage consisted of a transverse chain of five electrodes placed $5 \mathrm{~cm}$ above the inion and $5 \mathrm{~cm}$ apart, so that two electrodes were situated 5 and $10 \mathrm{~cm}$ to either side of a midline electrode. All electrodes were referred to a common midfrontal 
reference placed $12 \mathrm{~cm}$ above the nasion in the midline.

The stimulus used was a black-and-white checkerboard pattern made up of 50 minute squares projected on to a circular screen which, for full-field stimulation, subtended a radius of 16 degrees from a central fixation point when viewed from a distance of $1 \mathrm{~m}$. For half-field stimulation the screen was masked to produce a semicircular field subtending 16 degrees from the fixation point, which was then situated at the centre of the vertical margin of the field. The brightness levels were $227 \mathrm{~cd} / \mathrm{m}^{2}$ for the white and $8 \cdot 2 \mathrm{~cd} / \mathrm{m}^{2}$ for the black squares. Pattern reversal was produced once every $600 \mathrm{~ms}$ by a rapid lateral displacement of the pattern through one square width, the transition being completed in approximately $10 \mathrm{~ms}$. The occipital potentials were averaged using a PDP-12 computer which was triggered by the pattern reversal, the average response to 200 reversals being recorded in each run. The technique for recording in patients was unchanged except that eccentric fixation was used in some cases with 'macula-splitting' field defects to avoid the effects of a 'pseudofovea'.

\section{Results}

HALF-FIELD STIMULATION IN HEALTHY SUBJECTS The responses from the nine healthy persons all showed features that were essentially similar to those shown by the group average result (Fig. 1). In all subjects the large amplitude surface positive wave with a mean latency of $105 \mathrm{~ms}(\overline{\mathrm{P} 100})$ was recorded maximally from the electrodes in the midline and ipsilateral to the half field stimulated, the distribution of this component thus being largely responsible for the consistently asymmetrical half-field response. The ipsilateral waveform was further characterised by two surface negative waves $(\overline{\mathrm{N} 75}, \overline{\mathrm{N} 145})$ which immediately preceded and followed the positive wave, the whole NPN complex being widespread over the ipsilateral scalp. A steep voltage gradient was seen near the midline and the response recorded at the contralateral electrodes varied from small amplitude deflections, which were too near the background noise level to be clearly defined, to a triphasic PNP complex which was often best seen in the most lateral $(10-\mathrm{cm})$ channel. The major components of this complex appeared to be at similar latency but of opposite polarity to the ipsilateral components (Fig. 1). There was a considerable subject-to-subject variation in the presence and relative amplitude of these

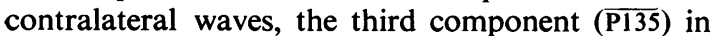
particular tending to dominate the contralateral response in an occasional individual. In such a

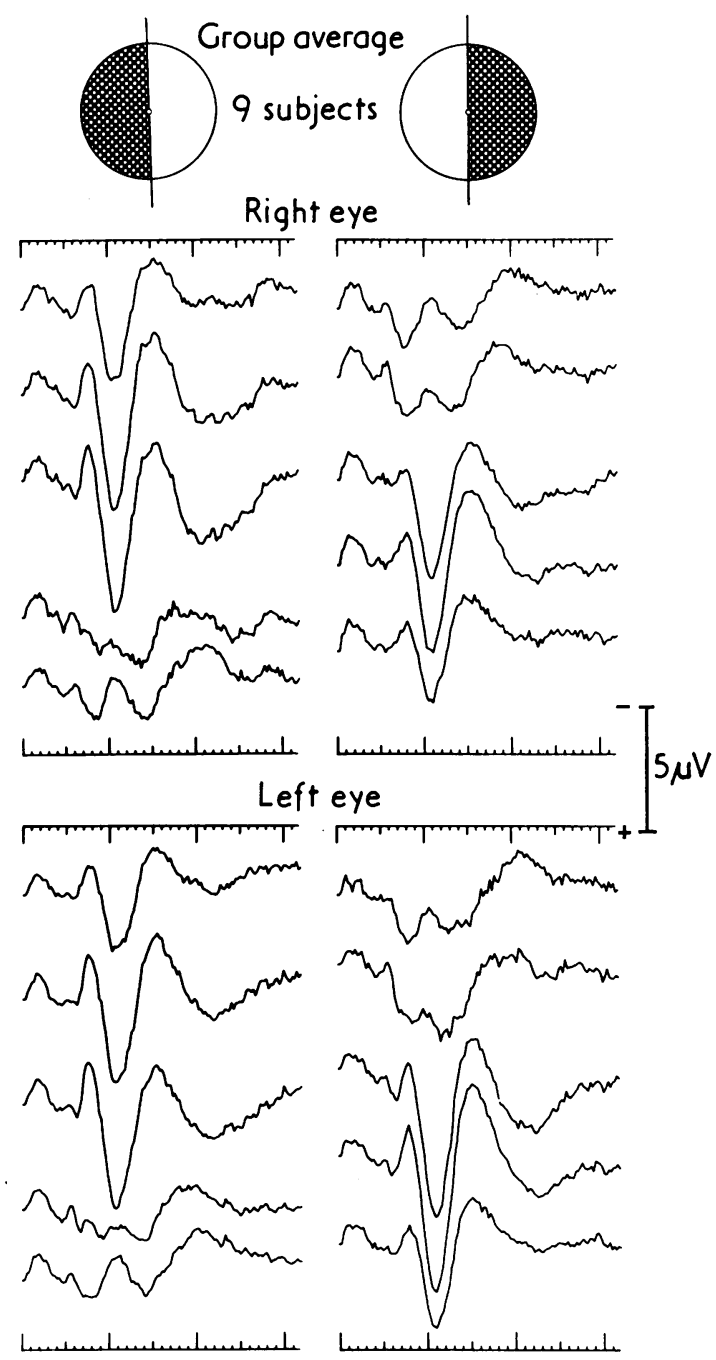

Fig. 1 Group average of monocular half-field responses of nine healthy subjects. Stimulated half fields shown checkered. Channels from top to bottom represent a transverse chain of five electrodes placed symmetrically at 5-cm intervals from left to right across occipital scalp (see head diagrams Figs. 2-5). Time scale for this and all other figures, 10, 50, $100 \mathrm{~ms}$ marks

case it is important not to confuse this contralateral positive component, which has a distinctly different latency and distribution, with the ipsilateral $\overline{\mathbf{P 1 0 0}}$.

The study of the normal half-field response in the nine subjects reported in this paper has been subsequently confirmed in 32 healthy subjects, all of whom show essentially similar features. A more detailed account of the relationships between the ipsilateral and contralateral responses evoked by 
half-field stimulation is in preparation (Blumhardt et al., 1977b).

FULL-FIELD STIMULATION IN HEALTHY SUBJECTS When full-field stimulation is performed using the same electrode montage, clearly the recorded responses must be a complex of the responses from both hemispheres. In our healthy subjects there was no consistent lateralisation of the full-field response related to the eye stimulated, the major components usually being fairly symmetrically distributed about the midline. This would be predicted from the usually similar amplitudes of the major components of the left and right half-field responses. Where these amplitudes were dissimilar on the two sides of the scalp minor full-field asymmetries were seen. These asymmetries are the same for stimulation of each eye, indicating that they are most probably due to anatomical differences between hemispheres. A fuller account of these hemisphere differences is in preparation (Barrett et al., 1977).

From Fig. 1 it can be predicted that the addition of corresponding channels from each half-field response will produce a large amplitude response in the midline, since both half-field responses have a large amplitude positive component $(\overline{\mathrm{P} 100})$ in the midline channel. In the lateral channels the addition of components of opposite polarity and usually unequal amplitude will result in the voltage of the summated response falling off to either side of the midline-for example, the addition of the large ipsilateral $\overline{\mathrm{P} 100}$ from the left channels of the left half-field response, with the small negative wave $(\overline{\mathrm{N}} \overline{105})$ from the contralateral channels of the right half-field response, produces a resultant positive wave which is reduced in amplitude. When the corresponding components from each half field are of about the same amplitude the full-field response would appear predominantly in the midline channel while the lateral channels will be relatively flat. This theoretical prediction from the algebraic summation of the half-field responses closely approximates the actual responses recorded from full-field stimulation in healthy subjects, an example of which is shown in Fig. 2.
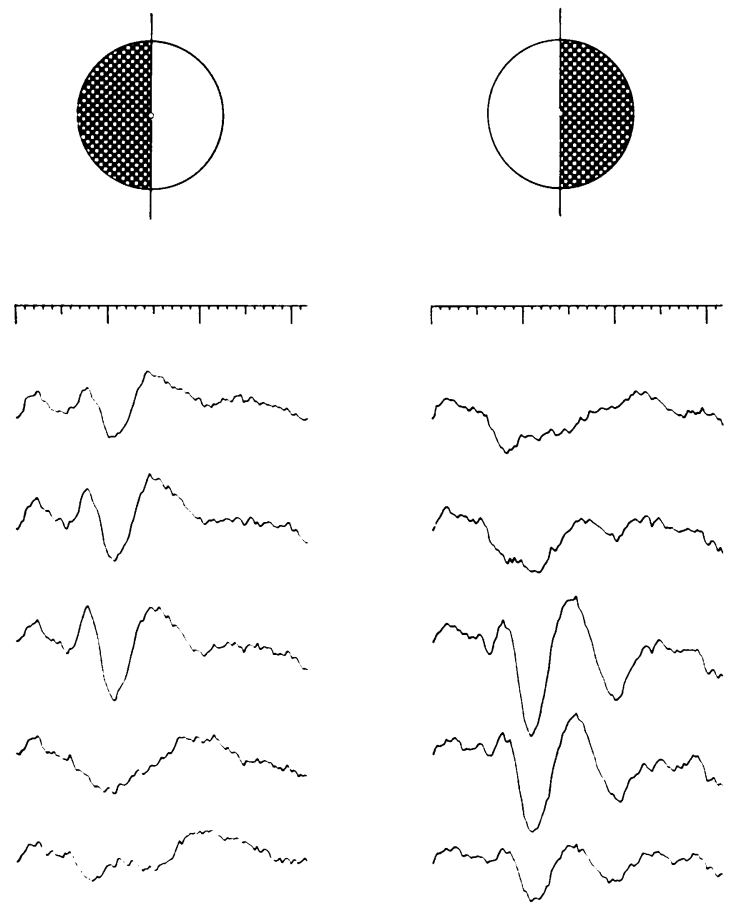

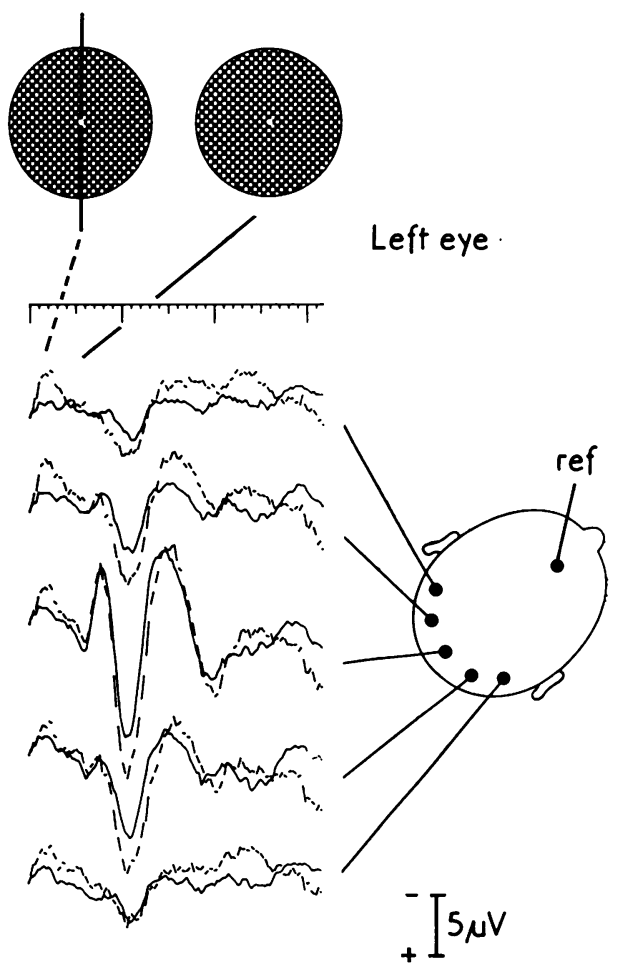

Fig. 2 Comparison of monocular full-field response with summated half-field responses in one healthy subject. The asymmetric left and right half-field responses are shown on left; the algebraic summation of these two responses is shown on right (dotted line) superimposed on separately recorded full-field response (solid line) 
ASYMMETRIES IN PATIENTS WITH HEMIANOPIC FIELD DEFECTS

\section{Homonymous hemianopia}

The results of stimulating a patient with a left homonymous hemianopia are shown in Fig. 3. This case was recorded after surgical removal of an arteriovenous malformation at the right occipital pole. The resulting left half-field defect was a dense homonymous 'macula-splitting' scotoma which extended out from the fixation point to about 25 degrees. Our 0 to 16 degree checkerboard stimulus was thus well within the scotoma to the left of the midline. Fullfield stimulation out to 16 degrees could therefore produce a response only from the preserved right half-field. Monocular full-field stimulation resulted in an asymmetric response which is the same for each eye. The typical ipsilateral complex (NPN) of the normal right half-field response is clearly seen in the midline and right-sided channels, while contralaterally the channel $5 \mathrm{~cm}$ from the midline shows only a small positive deflection and the most lateral channel is flat. Half-field stimulation, in this case using 2 degree eccentric fixation to allow for a pseudofovea, confirms the defect in two waysfirstly, by demonstrating that there is no response from the affected half-field and, secondly, by the similarity between the full-field and intact half-field responses (Fig. 4). The reduced size of the response from the right half-field, as compared with that in the full-field response, is no doubt a consequence of the reduction of the stimulus area adjoining the vertical meridian associated with the eccentric fixation. This type of asymmetry, which is the same for stimulation of each eye, we have called an 'uncrossed' asymmetry (Barrett et al., 1976a; Halliday et al., 1976).

\section{Bitemporal hemianopia}

In patients with bitemporal field defects the asymmetry of the full-field response is reversed for each eye, as shown by the records in Fig. 5. In the response from the left eye the major positive component

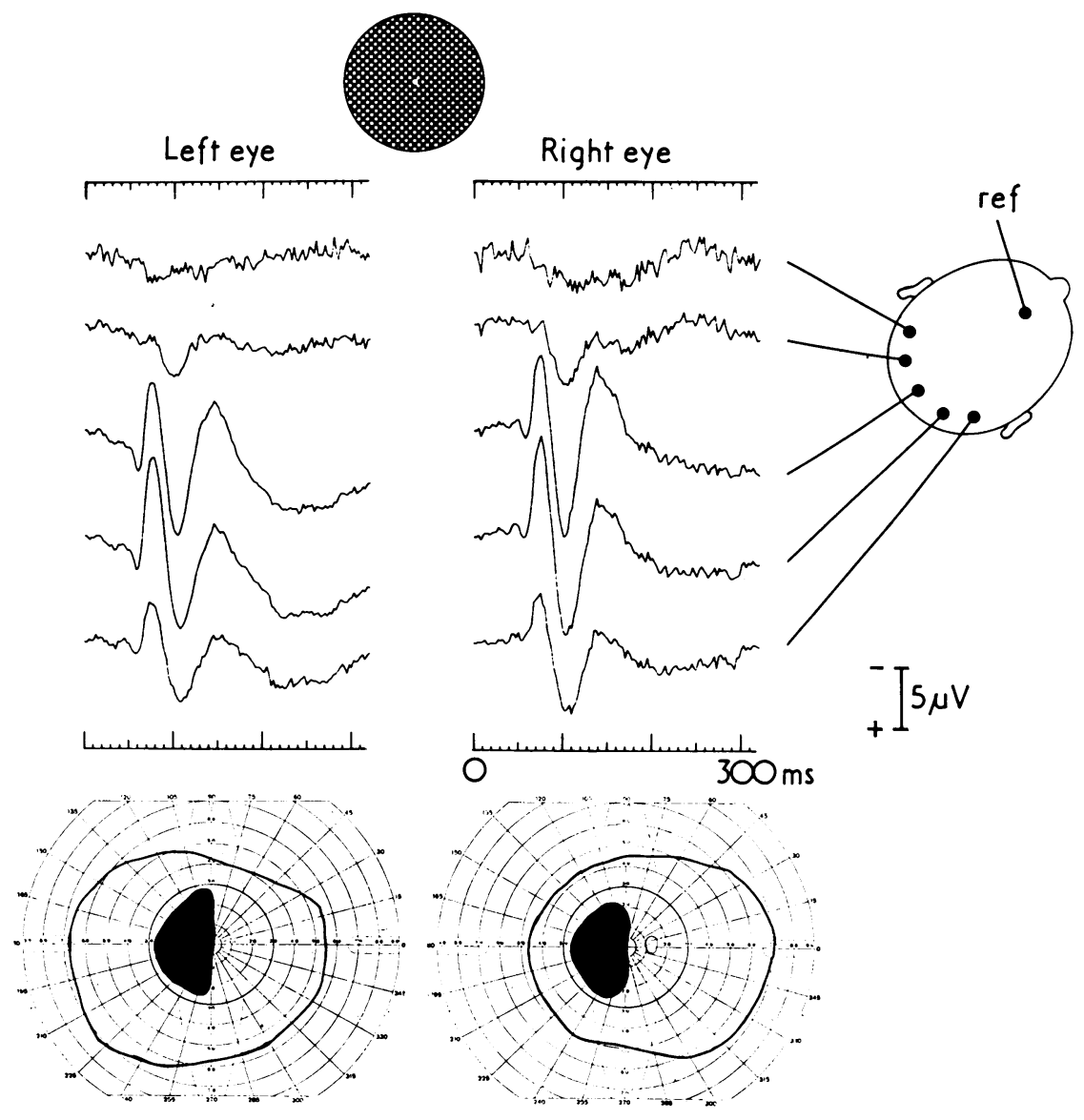

Fig. 3 Monocular full-field stimulation of patient after subtotal right occipital lobectomy. Goldmann visual fields show total homonymous 'maculasplitting' scotoma extending to about 25 degrees radius. Note that the asymmetry of the response is the same on stimulation of each eye-that is, it is 'uncrossed' 


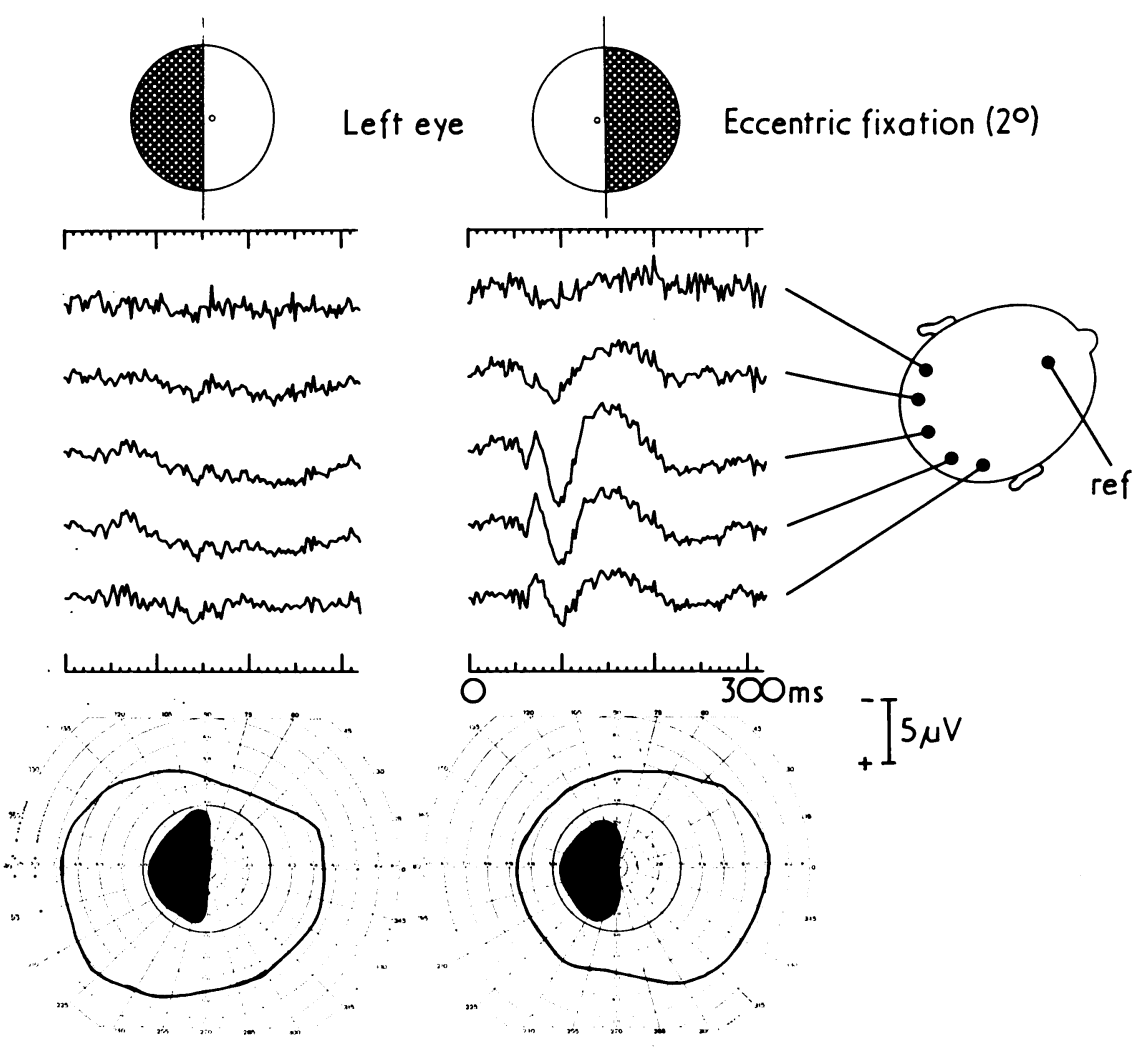

Fig. 4. Half-field stimulation using two degree eccentric fixation in patient whose full-field responses are shown in Fig. 3. Pattern-reversal within the scotomatous field defect produces no response detectable above the background noise (left hand record). Pattern-reversal within the preserved right half field produces $a$ typical ipsilateral complex' (NPN) from the scalp overlying the hemisphere with the surgical defect. Channels over intact left hemisphere are relatively flat. The smaller amplitude response when compared with that from full-field stimulation is due to eccentric fixation

( $\overline{\mathrm{P} 100})$ is clearly seen in the right-sided and midline channels - that is, from the scalp ipsilateral to the intact visual half field-while in the left-sided channels the $\overline{\mathrm{P} 100}$ has been replaced by a complex of opposite polarity with, in this case, a particularly prominent second positive wave. The absence of an ipsilateral response, particularly the $\overline{\mathrm{P} 100}$ from the left-sided channels, indicates that the left visual half field is defective. For the right eye the distribution of these components is reversed with the ipsilateral $\overline{\text { P100 }}$ being seen over the left side of the scalp accompanied by a PNP complex contralaterally. This type of asymmetry, which reverses on full-field stimulation of each eye, we have called a 'crossed' asymmetry (Barrett et al., 1976a; Halliday et al., 1976).

\section{Discussion}

A feature of many previous studies of patients with hemianopic field defects, including recent reports of the use of pattern stimuli by Wildberger et al. (1976), and Celesia and Daly (1976), has been the assumption that the decreased response should be recorded from the scalp electrodes situated contra- lateral to the visual field defect. This implies that the maximal, or at least optimal, response to stimulation of a normal half field will also be recorded from the contralateral electrodes, but no detailed description of the transverse distribution of full- or half-field responses in normal subjects had been reported in these papers. Our own studies show that this implication is not confirmed by the experimental findings, the major positivity being recorded ipsilaterally (Barrett et al., 1976a). These conclusions are confirmed by an examination of the records in the recently published report by Shagass et al. (1976), although owing to the much lower intensity of their pattern stimulus the $\overline{\mathrm{P} 100}$ component there appears as a $\overline{\mathrm{P} 125}$ wave.

With the use of a distant midline reference and 'monopolar' recording we have found that all normal half fields tested to date produce a clear positive wave at about $100 \mathrm{~ms}$ which is transversely distributed over at least $10 \mathrm{~cm}$ of the ipsilateral scalp (Barrett et al., 1976a). In a few individuals the $\overline{\text { P100 }}$ of the half-field response may spread slightly across the midline, but it invariably attains its maximum amplitude in the midline or ipsilateral channels. Together with the two surface negative waves 


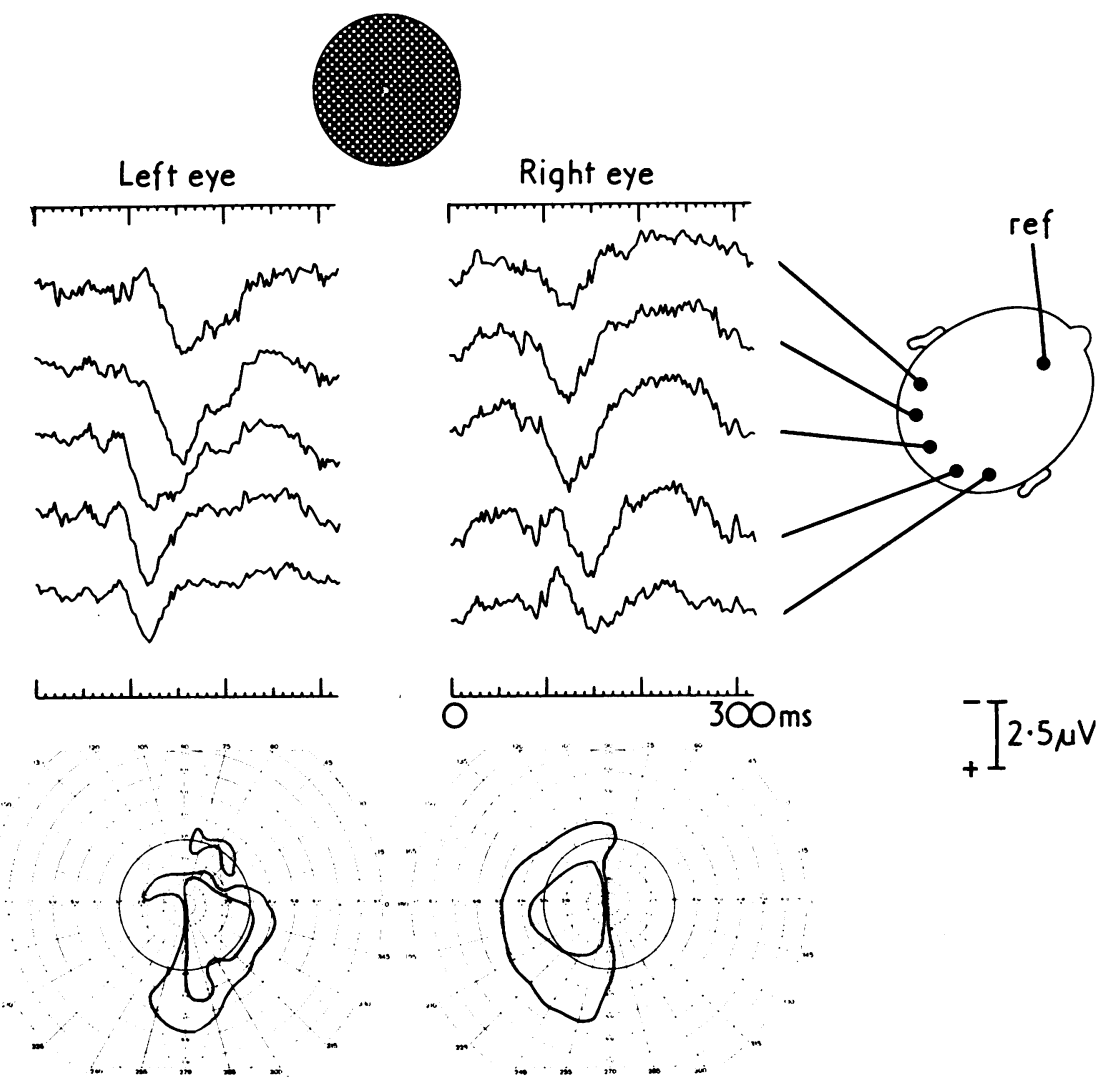

Fig. 5 Example of $a$ 'crossed' asymmetry recorded from patient with chiasmal compression due to pituitary adenoma. Goldmann perimetry showed irregular bitemporal hemianopia. Note that the positive wave in the contralateral channels, which is particularly prominent in the response from the left eye, is the contralateral component

( $\overline{P 135}$ ) seen in the half-field responses of normal subjects. This wave should not be confused with the ipsilateral positivity ( $\overline{P 100})$

immediately preceding and following the positivity, which are slightly less consistent, this positive wave makes up an ipsilateral triphasic complex which provides a useful indicator of the integrity of the visual half field. By contrast, the activity recorded from the scalp contralateral to the hadf field stimulated appears more variable, with components which are often small and sometimes absent or hidden, and of opposite polarity to the corresponding ipsilateral waves at similar latency.

We have proposed that the unexpected lateralisation of the major positive component $(\overline{\mathrm{P} 100})$ is related to the mesial location of the response generators on the hemisphere contralateral to the field stimulated, the ipsilateral electrodes being optimally placed to record a response from the posteromedial aspect of the contralateral hemisphere (Barrett et al., 1976a). The possiblity remained, however, that part of the response could be generated in the ipsilateral hemisphere, perhaps through the operation of the transcallosal connections (Beauchamp et al., 1976). This idea is not supported by the case demonstrated in Fig. 3. The normal amplitude $\overline{\mathrm{P} 100}$ which forms part of a typical 'ipsilateral complex' (NPN) is recorded from the channels directly over the hemisphere from which the tip of the occipital lobe has been excised. Hence it is unlikely to be generated on this side. Stimulation of the intact hemisphere, using 2 degree eccentric fixation to avoid stimulating the area of the field which may be expected to have transcallosal connections, clearly shows that the characteristic NPN complex is still recorded from the scalp ipsilateral to the half field stimulated (Fig. 4). These results indicate that although the $\overline{\mathrm{P} 100}$ is recorded in the midline and ipsilateral channels it is generated in the hemisphere contralateral to the half field stimulated, in agreement with our previous suggestion (Barrett et al., 1976a). Thus we have no evidence to support the proposal of Wildberger et al. (1976), who recorded a small ipsilateral steady-state response to an $8-\mathrm{Hz}$ pattern-reversal stimulus in one half field and attributed it to transmission by intercallosal connections. Further, from recordings made on patients with more extensive occipital lobectomies (Blumhardt et al., unpublished) it is clear that both the ipsilateral complex (NPN) and contralateral activity (PNP), while being recorded from opposite 
sides of the scalp, are generated in the hemisphere contralateral to the half field stimulated.

We have previously emphasised the critical importance of the montage and choice of reference electrode in this type of topographic study (Michael and Halliday, 1971; Barrett et al., 1976b). For example, the use of a transverse chain with bipolar linkages not only results in a loss of information when component amplitudes are similar at each electrode in the chain but, more seriously, can cause erroneous localisation of the maximal response (Barrett et al., 1976a; Shagass et al., 1976). The use of a reference placed to one side of the midline, as adopted by some workers in this field (Jeffreys and Axford, 1972a; 1972b; Wildberger et al., 1976) is inappropriate for the study of the transverse distribution of the asymmetric half-field responses. Since the ipsilateral response is widespread over the scalp to one side of the midline the ear reference is far from indifferent and will tend to exaggerate or reduce asymmetries on opposite sides depending on which hemisphere is stimulated. For this reason an ear reference is an unwise choice for the detection of field defects.

The placement of the occipital recording electrodes is also critical. The midline of the occipital scalp is an area of rapid voltage change. Recordings made from electrodes in or near the midline will therefore exhibit large differences in waveform with small changes in location on the scalp, and are thus unlikely to provide accurate information about hemianopic field defects unless augmented by information from more laterally placed electrodes. Asselman et al. (1975) found normal latency pattern evoked responses in three cases with hemianopia due to optic radiation lesions. Their conclusion that a macula-splitting hemianopia will not affect the latency recorded at a midline electrode is based on insufficient information, as the midline channel may be distorted in hemianopia or in the half-field response in normal subjects (Blumhardt et al., 1977b). Celesia and Daly (1976), using a combined technique of both flash and pattern-reversal with the 10-20 system of electrode placement, also found normal visual evoked responses in five patients with hemianopia. We do not consider that the commonly used $0_{1}$ and $0_{2}$ electrodes of the 10-20 system are suitable for the study of visual field defects, because they are situated too close to the midline. These studies emphasise that a multichannel recording of the distribution and waveform of the response over the lateral scalp rather than isolated amplitude and latency measurements is essential for accurate confirmation of pathological field defects.

The use of a circular stimulating field with central fixation necessarily produces a response which is a summation of the complexes produced by the two hemispheres. The two highly asymmetric halffield responses, when they are recorded separately and algebraically summated, closely approximate the response to full-field stimulation (Fig. 2). Thus a defect of one half field should 'unmask' the asymmetry of the other intact half-field response (NPN) which will then dominate the record, either flat channels or the triphasic complex of opposite polarity (PNP) indicating the side of the defective field. Two major types of asymmetry have emerged-namely, 'uncrossed' and 'crossed' - indicating homonymous and heteronymous hemianopias respectively. In dealing with homonymous hemianopias it is important to recognise the anatomical asymmetries that may occur in a proportion of healthy individuals. If full-field stimulation is used as a screening test these minor asymmetries can then be further tested with half-field stimulation. With this technique we have not encountered any difficulty in separating dense hemianopics from normals. In this respect the pattern stimulus marks a major advance on flash stimulation. With a 'crossed' asymmetry, which does not occur in normal individuals, one does not have to contend with the hemisphere differences and any record showing these features is strongly suggestive of a chiasmal lesion. On at least two occasions we have been able to indicate chiasmal compression in patients with gross reduction of visual acuity, ambiguous perimetry, and negative EMI scans in whom subsequent contrast studies have shown compressive spaceoccupying lesions. While this technique is accurate in recognising dense hemianopic visual field defects there is also some evidence from our recordings on patients to suggest that the method may be more sensitive than routine perimetry in detecting more subtle lesions of the visual pathways. In the published study of compressive lesions of the anterior visual pathways, for instance, two patients showed asymmetric responses without any associated field defect being demonstrable (Halliday et al., 1976).

\section{References}

Asselman, P., Chadwick, D. W., and Marsden, C. D. (1975). Visual evoked responses in the diagnosis and management of patients suspected of multiple sclerosis. Brain, 98, 261-282.

Barrett, G., Blumhardt, L. D., and Halliday, A. M. (1977). The paradoxical lateralisation of the evoked response to pattern-reversal stimulation of the visual half fields in man. In preparation.

Barrett, G., Blumhardt, L. D., Halliday, A. M., Halliday, Elise, and Kriss, A. (1976a). A paradox in the lateralisation of the visual evoked response. Nature, 261, 253-255.

Barrett, G., Blumhardt, L. D., Halliday, A. M., Halliday, Elise, and Kriss, A. (1976b). Paradoxical reversal of lateralisation of the half-field pattern evoked response 
with monopolar and bipolar electrode montages. Journal of Physiology, 258, 63-64P.

Beauchamp, M., Matthews, W. B., Small, D., and Stein, J. F. (1976). The topography of the visual evoked response to half-field stimulation. Journal of Physiology, 260, 46-47P.

Blumhardt, L. D., Barrett, G., Halliday, A. M., and Kriss, A. (1977a). The contralateral negativity of the half-field response and its association with central scotomata. Electroencephalography and clinical Neurophysiology. In press.

Blumhardt, L. D., Barrett, G., Halliday, A. M., and Kriss, A. (1977b). The effect of physiological 'scotomata' on the ipsilateral and contralateral responses to pattern reversal in one half-field. In preparation.

Celesia, G. G., and Daly, R. F. (1976). VECA: a new electrophysiological test for the diagnosis of optic nerve lesions. Neurology. In press.

Cobb, W. A., and Morton, H. B. (1970). Evoked potentials from the human scalp to visual half-field stimulation. Journal of Physiology, 208, 39-40P.

Halliday, A. M., McDonald, W. I., and Mushin, Joan (1972). Delayed visual evoked response in optic neuritis. Lancet, 1, 982-985.

Halliday A. M., McDonald, W. I., and Mushin, Joan (1973). Visual evoked response in diagnosis of multiple sclerosis. British Medical Journal, 4, 661-664.

Halliday, A. M., Halliday, Elise, Kriss, A., McDonald, W. I., and Mushin, Joan (1976). The pattern-evoked potential in compression of the anterior visual pathways. Brain, 99, 357-374.

Jeffreys, D. A., and Axford, J. G. (1972a). Source locations of pattern-specific components of human visual evoked potentials. I: Component of striate cortical origin. Experimental Brain Research, 16, 1-21.

Jeffreys, D. A., and Axford, J. G. (1972b). Source locations of pattern-specific components of human visual evoked potentials. II: Component of extrastriate cortical origin. Experimental Brain Research, 16, 22-40.

Kooi, K. A., Güvener, A. M., and Bagchi, B. K. (1965). Visual evoked responses in lesions of the higher optic pathways. Neurology, 15, 841-854.

Michael, W. F., and Halliday, A. M. (1971). Differences between the occipital distribution of upper and lower field pattern-evoked responses in man. Brain Research, 32, 311-324.

Oosterhuis, H. J. G. H., Ponsen, L., Jonkman, E. J., and Magnus, O. (1969). The average visual response in patients with cerebrovascular disease. Electroencephalography and Clinical Neurophysiology, 27, 23-34.

Shagass, C., Amadeo, M., and Roemer, R. A. (1976). Spatial distribution of potentials evoked by half-field pattern-reversal and pattern-onset stimuli. Electroencephalography and Clinical Neurophysiology, 41, 609-622.

Vaughan, H. G., Katzman, R., and Taylor, J. (1963). Alterations of visual evoked response in the presence of homonymous visual defects. Electroencephalography and Clinical Neurophysiology, 15, 737-746.

Wildberger, H. G. H., Van Lith, G. H. M., Wijngaarde, R., and Mak, G. T. M. (1976). Visual evoked cortical potentials in the evaluation of homonymous and bitemporal visual field defects. British Journal of Ophthalmology, 60, 273-278. 